\title{
Graphene oxide reinforced high surface area silica aerogels
}

\author{
Saoirse Dervin ${ }^{\mathrm{a}, \mathrm{b}}$, Yvonne Lang ${ }^{\mathrm{a}, \mathrm{c}}$, Tatiana Perova ${ }^{\mathrm{d}, \mathrm{e}}$, Steven H. Hinder ${ }^{\mathrm{f}}$, Suresh C. Pillai ${ }^{\mathrm{a}, \mathrm{b}, *}$ \\ a Nanotechnology \& Bio-Engineering Research Group, Department of Environmental Science, School of Science, Institute of Technology Sligo, Ash Lane, Sligo, Ireland \\ b Centre for Precision Engineering, Materials and Manufacturing Research (PEM), Institute of Technology Sligo, Ash Lane, Sligo, Ireland \\ c CERIS, School of Science, Institute of Technology Sligo, Sligo, Ireland \\ d Department of Electronic and Electrical Engineering, Trinity College Dublin, Dublin 2, Ireland \\ e ITMO University, 49 Kronverkskiy pr., Saint Petersburg, Russia \\ ${ }^{\mathrm{f}}$ The Surface Analysis Laboratory, Faculty of Engineering \&' Physical Sciences, University of Surrey, Guildford, Surrey GU2 7XH, UK
}

\section{A R T I C L E I N F O}

\section{Article history:}

Received 7 February 2017

Received in revised form 16 March 2017

Accepted 20 March 2017

Available online xxxx

\section{Keywords:}

Graphene

Graphene oxide

Silica

Aerogel

\begin{abstract}
A B S T R A C T
Silica aerogel structures were intercalated with graphene oxide (GO) via the addition of GO to the colloidal silica sol and subsequent sol-gel polymerization. The potential of GO to act as a nanofiller, for ambient pressure dried, hydrophobic silica aerogels, was systematically investigated. The influences of 0 to $2 \mathrm{wt} \% \mathrm{GO}$ loadings, on the physical properties of silica aerogels, were analysed by examining the bulk density, volume shrinkage (\%), pore volume and surface area of the composite aerogels. Additionally, the chemical composition of the composite gels was determined using FTIR, Raman, XRD and XPS. The study revealed that a GO addition of as little as $0.5 \mathrm{wt} \%$ is capable of supporting the porous framework of silica aerogels and also enhancing the properties of the gels simultaneously. The additions of $0.5 \mathrm{wt} \% \mathrm{GO}$ increased the surface area and pore volume of the aerogel from 390 to $700 \mathrm{~m}^{2} / \mathrm{g}$ and 0.59 to $0.99 \mathrm{~cm}^{3}$, respectively, and decreased aerogel density from 0.19 to $0.14 \mathrm{~g} / \mathrm{cm}^{3}$. The investigation therefore revealed that intercalation of the silica aerogel matrix with small quantities of $\mathrm{GO}$ can inhibit volume shrinkage during drying without hindering the physical properties of silica aerogels.
\end{abstract}

(c) 2017 Elsevier B.V. All rights reserved.

\section{Introduction}

The remarkable features of silica aerogels including ultralow density $\left(\sim 0.003 \mathrm{~g} / \mathrm{cm}^{3}\right)$, high specific surface area $\left(500 \mathrm{~m}^{2} / \mathrm{g}\right.$ to $\left.1200 \mathrm{~m}^{2} / \mathrm{g}\right)$, high porosity (80-99.8\%), high thermal insulation values $(0.005 \mathrm{~W} / \mathrm{mK})$ and versatility have resulted in their application in a number of industrial fields including aerospace, ultrasonic sensing, gas sensing, waste removal, optics, energy storage, catalysis and insulation [1-3].

Traditionally silica aerogels were prepared via supercritical drying [4-6]. This route uses supercritical fluids, elevated temperature and pressure to remove the liquid component of a gel, in the absence of capillary stress and surface tension. Though once a favoured method, this technique is expensive, time consuming and often hazardous, rendering the process impractical for industrial use. In order to exploit aerogels commercially, cost effective drying routes are required. Consequently, many upsurges in aerogel research have focused on the determination of alternative preparation routes [7-23].

\footnotetext{
* Corresponding author at: Nanotechnology \& Bio-Engineering Research Group, Department of Environmental Science, School of Science, Institute of Technology Sligo, Ash Lane, Sligo, Ireland.

E-mail address: pillai.suresh@itsligo.ie (S.C. Pillai).
}

In recent times, ambient pressure drying (APD) of sodium silicate gels has been identified as a feasible approach for the production of silica aerogels [24,25]. This method, however, often yields fragile gels [26]. Solvent extraction under atmospheric pressure evokes lateral compressive stress amongst the gel network in order to redress the loss of pore liquid during drying [27]. Polar Si-OH functionalities, located on the surface of the gels, interact with adjacent chains and give rise to relentless condensation reactions, which continue after complete formation of the silica network $[27,28]$. The interaction between the subsequent surface silanol groups has an elastic effect on the inner surfaces of the porous walls comprising the gel network [29]. The adjoining walls of the pores are drawn towards one another and eventually cause the porous network to collapse [29]. This results in irreversible shrinkage of the aerogel network [28,29]. The consequential innate brittleness of the silica network often limits extensive application of these materials. Accordingly, the mechanical properties of the interconnected silica network must be improved for commercial applications.

Several measures to enhance the mechanical properties and circumvent shrinkage of the aerogel network have been explored [30]. Surface modification or silylation, is one technique which has proved effective in preparing mechanically amended silica aerogels [14]. The process uses organosilanes such as hexamethyldisiloxane (HMDSO), hexamethyldisilazane (HMDZ), methyltriethoxy-silane (MTES) and trimethylchlorosilane (TMCS), converting $\mathrm{Si}-\mathrm{OH}$ functionalities into 
alkyl or aryl radicals, creating hydrophobic aerogels and preventing further interaction of adjacent $\mathrm{Si}-\mathrm{OH}$ functionalities [31-35].

Alternatively several studies have integrated the gel network with composite materials, such as crosslinking polymers, nanoparticles and/or ceramic fibres [36-46]. Conversely, it is often difficult to improve the mechanical properties of silica gels with the use of nanofillers or cross-linking reagents without altering or hampering additional properties such as the bulk density and surface area $[36,47]$. However, with effective control of each stage of aerogel synthesis and composite integration, the incorporation of additional materials between the weaker neck regions of the silica nanoparticles enhances the strength of the gel network and can also yield aerogels with improved or additional properties [30].

Graphene oxide (GO) [48], has recently been regarded as an attractive nanofiller for 3D architectures due to its unique two-dimensional structure [49], and other attractive properties (fracture stress B = $63 \mathrm{GPa}$ ). However, little information regarding the effect of GO on the silica matrix is provided within current literature. Previous studies have demonstrated the successful incorporation of reduced graphene oxide (rGO) within the matrices of silica aerogels, however, to date, no systematic studies have explored the reinforcement of silica aerogels with GO [50]. Composite formation of GO intercalated silica aerogels concedes the possibility of preparing high surface area, porous materials with improved properties. Alternatively, incorporating GO amongst the silica network may increase the mechanical properties of the aerogel but may also hinder the material's properties.

The current study therefore aims to introduce GO into the matrix of silica aerogels to determine the potential of the carbonaceous material as a nanofiller for silica aerogels. The effect of GO loading on the density, pore volume/diameter and surface area of the composite gels was investigated.

\section{Experimental}

\subsection{Synthesis of graphite oxide (GtO)}

Graphite oxide (GtO) was prepared from natural graphite following an improved synthesis [51]. In a typical procedure, a solution containing $360 \mathrm{ml}$ concentrated $\mathrm{H}_{2} \mathrm{SO}_{4}$ and $40 \mathrm{ml}$ concentrated $\mathrm{H}_{3} \mathrm{PO}_{4}$ was added to $3 \mathrm{~g}$ of graphite flakes and $18 \mathrm{~g} \mathrm{KMnO}_{4}$. Once an emerald green homogenous mixture had been obtained the solution was heated to 50 ${ }^{\circ} \mathrm{C}$ and stirred for $12 \mathrm{~h}$. After observation of a green-brown colour change the solution was cooled to room temperature poured over $400 \mathrm{ml}$ deionised (DI) ice and $\mathrm{H}_{2} \mathrm{O}_{2}$ was subsequently added until a bright yellow colour was achieved. The obtained yellow mixture was allowed to cool before washing with water, $30 \% \mathrm{HCl}$ and ethanol, respectively via centrifugation at $10,000 \mathrm{rpm}$ for $20 \mathrm{~min}$. Finally, the obtained pellet was coagulated with diethyl ether and the resulting suspension was filtered over a PTFE membrane with a $0.45 \mu \mathrm{m}$ pore size. The solid substance obtained on the filter was dried overnight at ambient conditions.

\subsection{Preparation of silicic acid (S.A.) solution}

The source of silica used for the preparation of hydrogels was waterglass i.e. sodium silicate ( $\geq 27 \mathrm{wt} \%$ ) from Sigma Aldrich, Ireland. For the preparation of a $20 \mathrm{ml}$ composite sol $5.93 \mathrm{ml}$ sodium silicate solution was diluted with $7.035 \mathrm{ml}$ DI water. The dilute sodium silicate solution was passed through an ion-exchange resin (Amberlite ${ }^{\circledR}$ IR120 hydrogen form; Sigma Aldrich, Ireland) (Scheme 1) in order to remove the $\mathrm{Na}^{+}$ions and obtain a silicic acid solution with a $\mathrm{pH}$ of 2.5-3.0.

\subsection{Preparation of graphene oxide (GO) solution}

Various amounts of GtO were added to $7.04 \mathrm{ml}$ DI water. Typically, $41.21 \mathrm{mg}, 82.43 \mathrm{mg}$ and $164.85 \mathrm{mg}$ GtO were added to $7.04 \mathrm{ml} \mathrm{DI}$ water in order to prepare GO aqueous solutions which would account of a $0.5,1$ and $2 \mathrm{wt} \%$ loading in the silicic acid sol. The dispersed GtO sheets were then exfoliated via ultrasonication for $1 \mathrm{~h}$ in order to obtain an aqueous dispersion of GO.

\subsection{Preparation of GO-S.A. solution}

$\mathrm{GO}-\mathrm{SiO}_{2}$ composite aerogels were prepared by sol-gel polymerization of silicic acid sols containing different loadings of GO in the range of $0-2$ wt\% (scheme 1 ).

The weight varying GO solutions were added to each of the $20 \mathrm{ml} \mathrm{si}-$ licic acid solutions in order to obtain composite sols which contained various loadings of GO ranging from 0 to $2 \mathrm{wt} \%$ (weight percentage of the aerogel, assuming full conversion of silicic acid into $\mathrm{SiO}_{2}$ aerogel).

\section{5. $\mathrm{GO}-\mathrm{SiO}_{2}$ wet gel formation}

Concentrated $\mathrm{NH}_{4} \mathrm{OH}$ (typically one drop) was added to the pure $\mathrm{SiO}_{2}$ sol and the $\mathrm{GO}-\mathrm{SiO}_{2}$ sols until the $\mathrm{pH}$ of the sols reached $\mathrm{pH}$ 6-7. The sols were then kept at $50{ }^{\circ} \mathrm{C}$ for gelation. The obtained hydrogels were aged for $3 \mathrm{~h}$ at $50{ }^{\circ} \mathrm{C}$ to strengthen the silica network of the composite materials. The strengthened monoliths then underwent a $24 \mathrm{~h}$

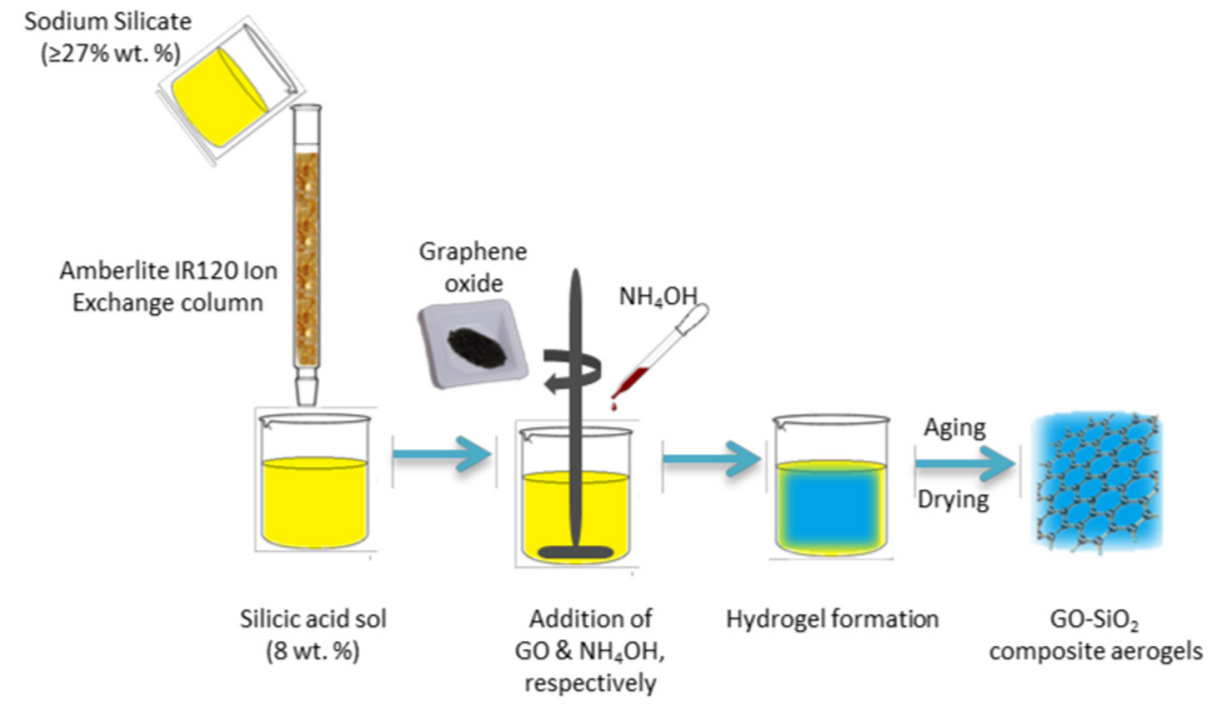

Scheme 1. Preparation of $\mathrm{GO}-\mathrm{SiO}_{2}$ composite aerogels. 
Table 1

The effects of $\mathrm{GO}$ loading on the textural properties of $\mathrm{SiO}_{2}$ aerogels.

\begin{tabular}{|c|c|c|c|c|c|c|}
\hline GO content (wt\%) & Gelation time (min) & Density $\left(\mathrm{g} / \mathrm{cm}^{3}\right)$ & Volume shrinkage (\%) & Surface area $\left(\mathrm{m}^{2} / \mathrm{g}\right)( \pm 5)$ & Pore volume $\left(\mathrm{cm}^{3} / \mathrm{g}\right)( \pm 0.05)$ & Pore diameter $(\mathrm{nm})( \pm 0.2)$ \\
\hline 0 & 10 & 0.19 & 80 & 390 & 0.59 & 5.56 \\
\hline 0.5 & 10 & 0.14 & 65 & 700 & 0.99 & 5.31 \\
\hline 1 & 10 & 0.17 & 69 & 708 & 0.97 & 5.19 \\
\hline 2 & 20 & 0.23 & 75 & 798 & 0.60 & 3.84 \\
\hline
\end{tabular}

solvent exchange in $\mathrm{MeOH}$ and subsequent surface modification. The gels were immersed in MeOH:TMCS:hexane in the volumetric ratio $1: 1: 2$ at room temperature for $24 \mathrm{~h}$. After surface modification the silylated gels were washed 4 times in $24 \mathrm{~h}$ with hexane to remove all

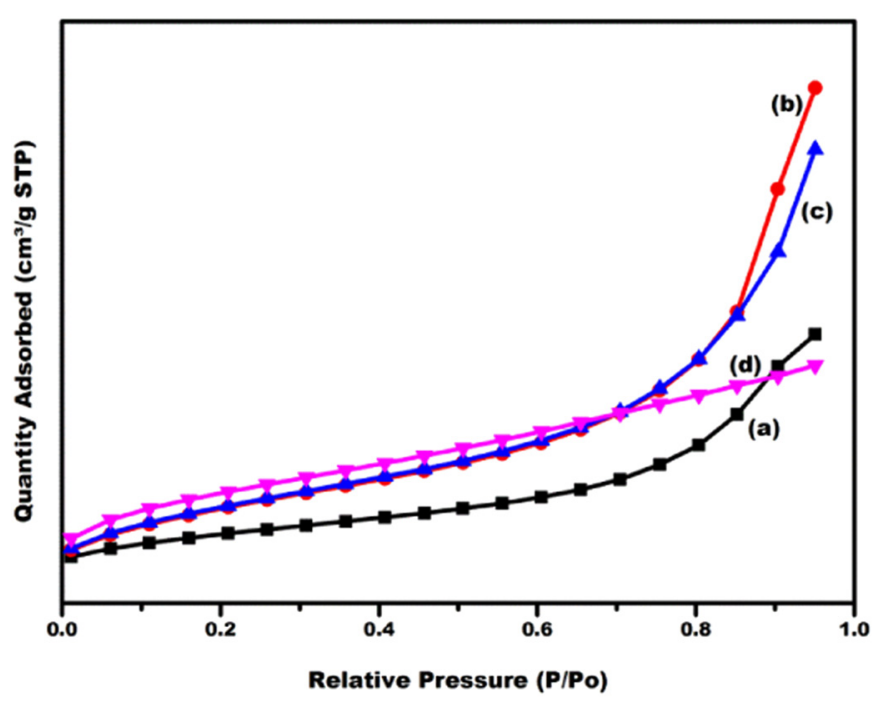

Fig. 1. Linear isotherm plots of (a) 0 , (b) 0.5 , (c) 1 and (d) $2 \mathrm{wt} \% \mathrm{GO}-\mathrm{SiO}_{2}$ composite aerogels. traces of TMCS before drying at $80^{\circ} \mathrm{C}$ for $5 \mathrm{~h}$, and at $150{ }^{\circ} \mathrm{C}$ for $1 \mathrm{~h}$. For a complete evaporation of the pore liquid, the gels were finally dried at $200{ }^{\circ} \mathrm{C}$ for an hour. The aerogel samples were allowed to cool to room temperature before being ground into a fine powder and characterized by various techniques (scheme 1 ).

\subsection{Characterisation}

The gelation time was the interval between the introduction of the gels moulds to the oven at $50{ }^{\circ} \mathrm{C}$ and the point at which the sol was no longer mobile. The bulk density of the aerogels was determined from their mass to volume ratio. A cylindrical column of known volume was filled with the aerogel powders and the bulk density $\left(d_{B a}\right)$ was calculated from its mass $\left(m_{a}\right)$ to volume $\left(V_{a}\right)$ ratio.

$d_{B a}=\frac{m_{a}}{V_{a}}$

The volume shrinkage $\left(V_{s}\right)$ of the aerogels were determined using the following formula:

$V s \%=\left(1-\frac{V a}{V g}\right) X 100$

where $V_{g}$ is the volume of hydrogel. The textural properties of the aerogels were investigated by standard $\mathrm{N}_{2}$ gas adsorption method using a Gemini VII 2390 Surface Area Analyzer (Micromeritics). The specific surface areas and pore size distributions were calculated by the BET
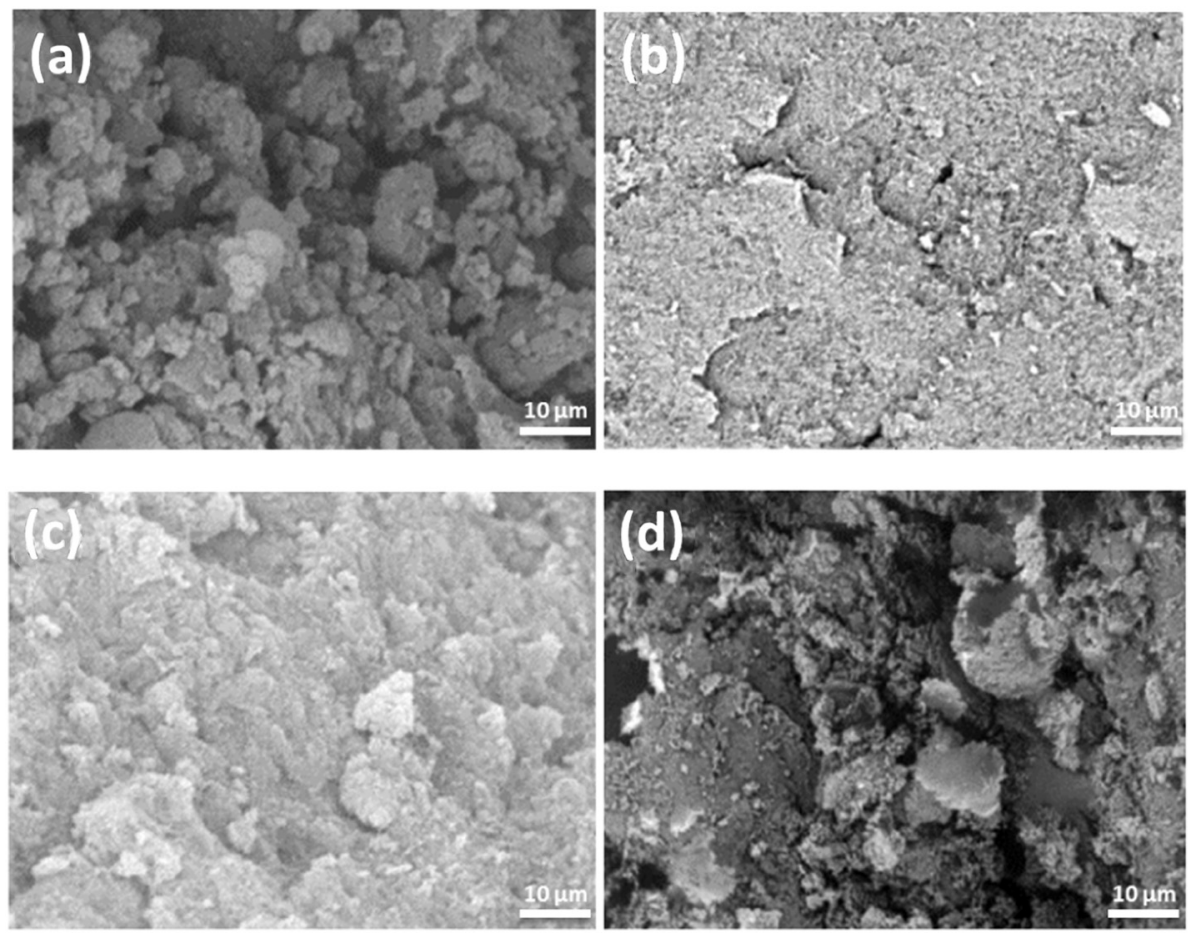

Fig. 2. SEM images of (a) 0 , (b) 0.5 , (c) 1 and (d) $2 \mathrm{wt} \% \mathrm{GO}-\mathrm{SiO}_{2}$ composite aerogels $\times 10,000$. 


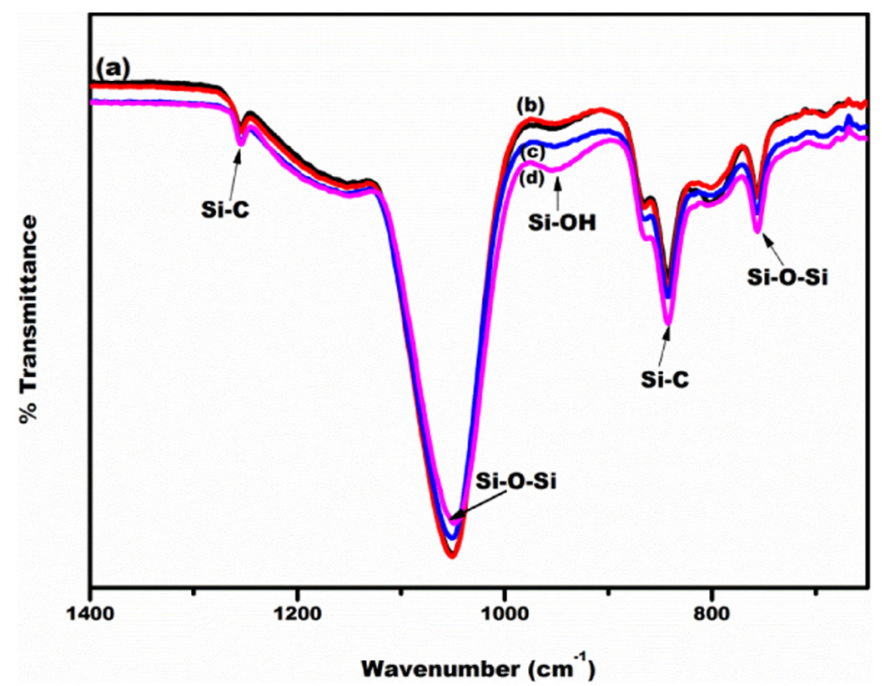

Fig. 3. FTIR spectra of (a) 0 (b) 0.5 (c) 1 and (d) 2 wt\% GO-SiO ${ }_{2}$ composite aerogels.

(Brunauer-Emmett-Teller) method. The aerogel powders were degassed at $300{ }^{\circ} \mathrm{C}$ for $3 \mathrm{~h}$ and the adsorption isotherms were obtained at $-196.15^{\circ} \mathrm{C}$. The morphologies of the aerogels were examined by scanning electron microscopy (Siemens TM1000). The extent of silica polymerization and the surface properties of the aerogels were investigated by means of Fourier Transform Infrared (FTIR) spectrometer Perkin Elmer Spectrum 100. The Raman scattering from composite gels was investigated using Renishaw RM1000 micro-Raman system, equipped with Leica microscope (using $50 \times$ objective lens), Peltier cooled CCD camera, $1800 \mathrm{l} / \mathrm{mm}$ grating, and He-Ne laser with excitation wavelength of $633 \mathrm{~nm}$. An XRD patterns were also obtained using a Siemens D500 X-ray powder diffractometer with a diffraction angle range of $2 \theta=10^{\circ}-80^{\circ}$ using $\mathrm{Cu} \mathrm{KR}$ radiation $(\lambda=0.15418 \mathrm{~nm}$.) The elemental composition of each of the composite gels was examined using $\mathrm{X}$-ray photoelectron spectroscopy (XPS).

XPS was conducted using a Thermo VG Scientific (East Grinstead, UK) ESCALAB Mk II spectrometer, inclusive of an XR3 twin anode X- ray source $(\mathrm{AlK} \alpha / \operatorname{MgK} \alpha)$ and an Alpha 110 analyzer. The twin anodes AlK $\alpha$ X-ray source $(h \nu=1486.6 \mathrm{eV})$ was operated at $300 \mathrm{~W}(15 \mathrm{kV}$ $\times 20 \mathrm{~mA}$ ). A pass energy of $200 \mathrm{eV}$ and a step size of $0.4 \mathrm{eV}$ were employed to obtain all survey spectra. In order to acquire C1s, O1s and $\mathrm{Si} 2 \mathrm{p}$ high resolution spectra a pass energy of $20 \mathrm{eV}$ and a step size of $0.2 \mathrm{eV}$ were used.

\section{Results and discussion}

The influence of GO loading on the physical and structural properties of the composite aerogels was investigated by varying the GO content. 0 , $0.5,1$ and 2 wt\% GO was added to each of the silicic acid sols. The composite aerogel with the greatest GO wt\% content ( $2 \mathrm{wt} \%$ ) required 20 min to form a rigid gel. On the contrary, hydrogels with a lower GO content, and the pure $\mathrm{SiO}_{2}$ gel, set within $10 \mathrm{~min}$. The retarded gelation of the $2 \mathrm{wt} \% \mathrm{GO}$ gel is presumably due to the saturated adsorption of GO sheets on the surface of and amongst the $\mathrm{SiO}_{2}$ particles. Thus, the hydrolysis and condensation reactions between siloxane chains were hindered and gelation delayed [52].

Due to drying related stresses, silica aerogels often tend to collapse and shrink during the final stages of synthesis [53]. This shrinkage results from compressive stresses endured by the solid $\mathrm{SiO}_{2}$ gel network [54]. These stresses which arise predominantly from surface and capillary tensions cause the porous network to cave and collapse. The former results in gel shrinkage and densification, producing an aerogel with low specific surface area, low pore volume and high density [55].

The present investigation attempted to avoid such shrinkage, and the consequential depreciation of the physical properties of silica aerogels, by examining the potential of $\mathrm{GO}$ as a reinforcing agent. A significant degree of shrinkage was observed for all of the prepared aerogels. However, the addition of GO reduced the degree of volume shrinkage by up to $19 \%$.

As the GO loading increased to $0.5 \mathrm{wt} \%$ the level of shrinkage decreased from 80 to $65 \%$ (Table 1), suggesting that the incorporation of GO particles amongst the silica network strengthened the gel structure. However, as the GO content further increased to $2 \mathrm{wt} \%$, a more notable degree of shrinkage was observed, indicating that small quantities of GO are sufficient to circumvent shrinkage during drying.

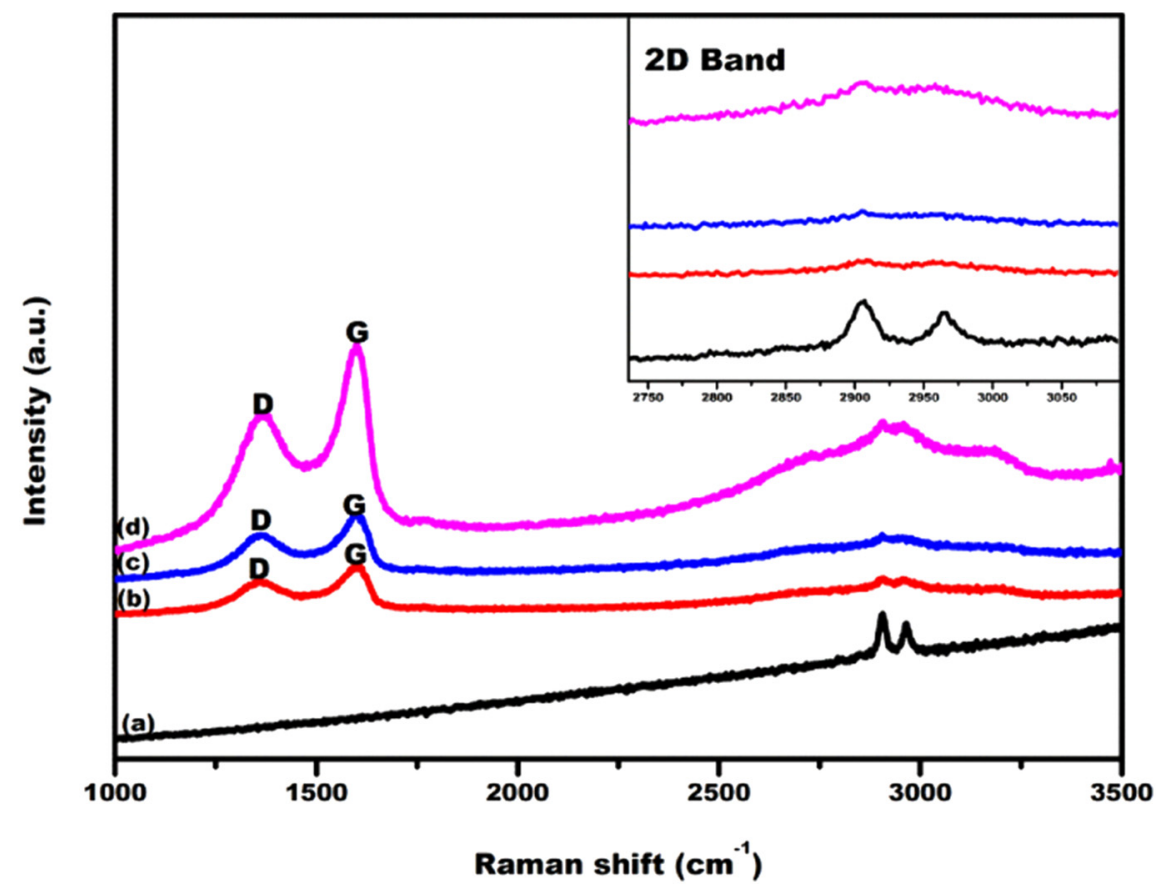

Fig. 4. Raman spectra of (a) 0 (b) 0.5 (c) 1 and (d) $2 \mathrm{wt} \% \mathrm{GO}-\mathrm{SiO}_{2}$ composite aerogels. 
The density of the GO intercalated gels, presented in Table 1, followed a similar trend to the degree of shrinkage witnessed for each of the gels. The 2 wt\% $\mathrm{GO}-\mathrm{SiO}_{2}$ aerogel exhibited a density of $0.23 \mathrm{~g} / \mathrm{cm}^{3}$, which is slightly denser than the pure $\mathrm{SiO}_{2}$ gel $\left(0.19 \mathrm{~g} / \mathrm{cm}^{3}\right)$. However, the addition of $0.5 \mathrm{wt} \% \mathrm{GO}$ reduced the aerogel density to $0.14 \mathrm{~g} / \mathrm{cm}^{3}$. The decrease in density proposes a lesser extent of pore collapse, suggesting that GO is capable of acting as a reinforcing agent for silica aerogels. Consequently, the density and pore volume of each of the composite gels are indirectly proportional to one another (Table 1). The $68 \%$ increase in pore volume again revealed that the addition of $0.5 \mathrm{wt} \% \mathrm{GO}$ prevented pore collapse when subjecting the composite gels to drying related stresses. The subsequent decrease in pore volume, upon the addition of $2 \mathrm{wt} \% \mathrm{GO}$, suggests an excess loading of GO within the silica matrix. The silica network has presumably become saturated with GO, increasing the occupancy of GO within the pore of the composite gels, thus reducing pore accessibility [56].

Fig. 1 shows the $\mathrm{N}_{2}$ adsorption isotherms of $0,0.5,1$ and 2 wt\% GO aerogels. The adsorption isotherms of the pure $\mathrm{SiO}_{2}$ aerogel and each of the GO intercalated aerogels correspond to type IV, which is consistent with mesoporous materials [57]. The isotherms presented by the $0-1 \mathrm{wt} \% \mathrm{GO}-\mathrm{SiO}_{2}$ aerogels indicate the presence of both mesopores $(2-50 \mathrm{~nm})$ and macropores $(>50 \mathrm{~nm})$. The existence of macropores $(>50 \mathrm{~nm})$ is evidenced by elevated adsorption at relative pressures above 0.90 and an unsaturated adsorption isotherm [58-60]. The isotherm of the $2 \mathrm{wt} \%$ GO gel however, persists in a horizontal manner which suggests that the materials is mesoporous and does not contain macropores [57]. Additionally, the average pore diameter decreases as the GO content increases to 2 wt\% (5.56-3.84) Table 1.

The surface morphology of the composite aerogels was investigated using SEM (Fig. 2). Each of the prepared aerogels possessed a rough structure which continued to coarsen as the GO loading increased. A more compact structure was obtained as GO loading increased due to the coalescence of the $\mathrm{SiO}_{2}$ and $\mathrm{GO}$ particles. The agglomeration of $\mathrm{GO}$ within the $\mathrm{SiO}_{2}$ network is also evidenced by the reduction of pore volume as GO loading approaches $2 \mathrm{wt} \%$.

The FTIR spectra of each of the aerogel composites are presented in Fig. 3. Slight variation corresponding to an increase of GO content can be observed from the spectra, however, the addition of GO did not dramatically alter $\mathrm{SiO}_{2}$ polymerization. The peaks at $1050 \mathrm{~cm}^{-1}, 950 \mathrm{~cm}^{-1}$ and $800 \mathrm{~cm}^{-1}$ are attributed to the $\mathrm{Si}-\mathrm{O}-\mathrm{Si}$ asymmetric stretching, $\mathrm{Si}-\mathrm{OH}$ and $\mathrm{Si}-\mathrm{O}-\mathrm{Si}$ bending, respectively $[61,62]$.

The $\mathrm{Si}-\mathrm{O}-\mathrm{Si}$ band at $1050 \mathrm{~cm}^{-1}$ begins to decrease in intensity and shifts to the low-frequency side as the GO content is increased. Similarly, the intensity of the Si-O-Si peak at $750 \mathrm{~cm}^{-1}$ also begins to decrease significantly.

The variations in peak intensity confirm that as the GO content increases from 0 to $2 \mathrm{wt} \%$ the number of free hydroxyl and hydrocarbon groups present on the surface of the composite gels decreases [63]. In contrast, the peak at $950 \mathrm{~cm}^{-1}$, associated with the $\mathrm{Si}-\mathrm{OH}$ vibrations, increases as the loading of GO increases to $2 \mathrm{wt} \%$, presumably due to the abundance of oxygen functional groups located on the edges of the $\mathrm{GO}$ sheets [64]. Furthermore, the $\mathrm{Si}-\mathrm{C}$ peak located at approximately $1250 \mathrm{~cm}^{-1}$ begins to intensify as GO content increases to $2 \mathrm{wt} \%$.

Raman spectra of the various $\mathrm{GO}-\mathrm{SiO}_{2}$ composite aerogels are presented in Fig. 4. The D, G and 2D bands associated with graphene based materials, [65,66] are observed around $1335 \mathrm{~cm}^{-1}, 1575 \mathrm{~cm}^{-1}$ and $2700 \mathrm{~m}^{-1}$, respectively. Furthermore, two sharp peaks are observed on the background of the broad 2D band of graphene at approximately 2887 and $2965 \mathrm{~cm}^{-1}$. The latter can be assigned to amorphous $\mathrm{SiO}_{2}$, due to the appearance and intensity of both the 2887 and $2965 \mathrm{~cm}^{-1}$ peak within the Raman spectrum of pure $\mathrm{SiO}_{2}$ aerogel (Fig. 4, line (a)). The intensity of the $\mathrm{SiO}_{2}$ peaks decreases as the $\mathrm{GO}$ content of the aerogel increases. Conversely, as the GO loading increases aerogel increases. Conversely, as the GO loading increases from 0 to $2 \mathrm{wt} \%$ the $\mathrm{D}$ and $\mathrm{G}$ peaks become more intense. The XRD patterns of the various wt\% $\mathrm{GO}-\mathrm{SiO}_{2}$ composites (ESI Fig. 1.), display a
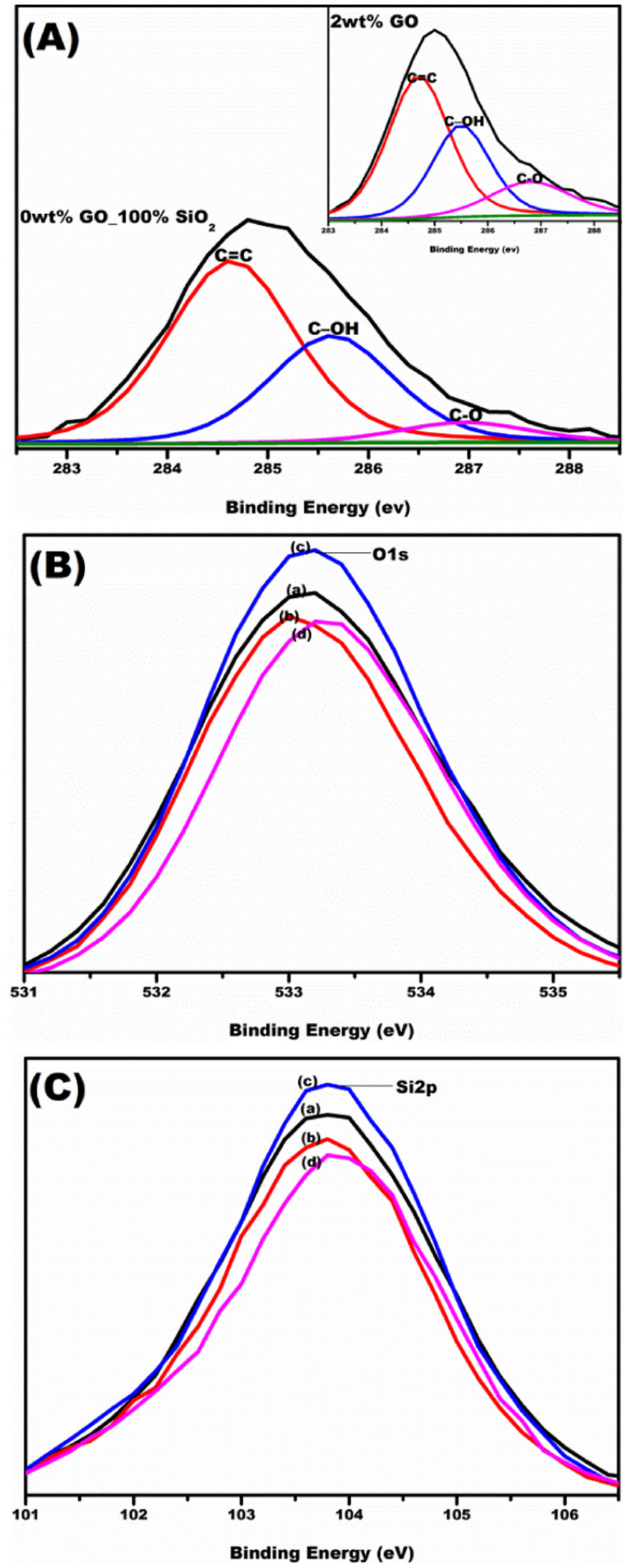

Fig. 5. (A) C1s XPS spectra of the $0 \mathrm{wt} \%$ and (inset) $2 \mathrm{wt} \% \mathrm{GO}-\mathrm{SiO}_{2}$ aerogels, (B) O1s XPS spectra of and (C) 01s XPS spectra of (a) 0, (b) 0.5, (c) 1 and (d) 2 wt\% GO-SiO 2 composite aerogels. 
Table 2

XPS peak positions and atomic \% $\mathrm{Si}, \mathrm{C}$, and $\mathrm{O}$ in $\mathrm{GO}-\mathrm{SiO}_{2}$ composite aerogels.

\begin{tabular}{|c|c|c|c|c|c|c|c|c|c|c|c|c|}
\hline \multirow[b]{2}{*}{ Element } & \multicolumn{3}{|l|}{$0 \mathrm{wt} \% \mathrm{GO}$} & \multicolumn{3}{|l|}{$0.5 \mathrm{wt} \% \mathrm{GO}$} & \multicolumn{3}{|l|}{$1 \mathrm{wt} \% \mathrm{GO}$} & \multicolumn{3}{|l|}{$2 \mathrm{wt} \% \mathrm{GO}$} \\
\hline & Binding energy & Bond & Atomic \% & Binding energy & Bond & Atomic \% & Binding energy & Bond & Atomic \% & Binding energy & Bond & Atomic \% \\
\hline \multirow[t]{3}{*}{$\mathrm{C} 1 \mathrm{~s}$} & 284.6 & $\mathrm{C}=\mathrm{C}$ & 8.78 & 284.6 & $C=C$ & 10.76 & 284.6 & $\mathrm{C}=\mathrm{C}$ & 9.57 & 284.7 & $\mathrm{C}=\mathrm{C}$ & 11.00 \\
\hline & 285.6 & $\mathrm{C}-\mathrm{OH}$ & 4.92 & 285.6 & $\mathrm{C}-\mathrm{OH}$ & 5.26 & 285.6 & $\mathrm{C}-\mathrm{OH}$ & 6.71 & 285.5 & $\mathrm{C}-\mathrm{OH}$ & 6.82 \\
\hline & 287.0 & $\mathrm{C}-\mathrm{O}$ & 0.96 & 286.8 & $\mathrm{C}-\mathrm{O}$ & 1.05 & 286.9 & $\mathrm{C}-\mathrm{O}$ & 1.71 & 286.8 & $\mathrm{C}-\mathrm{O}$ & 3.46 \\
\hline $01 \mathrm{~s}$ & 533.2 & $\mathrm{C}-\mathrm{OH}$ & 61.47 & 533.1 & $\mathrm{C}-\mathrm{OH}$ & 59.29 & 533.2 & $\mathrm{C}-\mathrm{OH}$ & 59.04 & 533.3 & $\mathrm{C}-\mathrm{OH}$ & 56.63 \\
\hline Si2p & 103.7 & $\mathrm{SiO}_{2}\left(\mathrm{Si}^{4+}\right)$ & 23.86 & 103.8 & $\mathrm{SiO}_{2}\left(\mathrm{Si}^{4+}\right)$ & 23.78 & 103.9 & $\mathrm{SiO}_{2}\left(\mathrm{Si}^{4+}\right)$ & 23.01 & 103.9 & $\mathrm{SiO}_{2}\left(\mathrm{Si}^{4+}\right)$ & 22.21 \\
\hline
\end{tabular}

characteristic GO peak, which appears at approximately $2 \theta=10^{\circ}$, [67] which may be due to the small amounts of $\mathrm{GO}$ added to the $\mathrm{SiO}_{2}$ samples. Furthermore, the disappearance of the $2 \theta=10^{\circ}$ peak suggests diminishing of long-range structure ordering of the graphene domains, [68] which illustrates the interaction of $\mathrm{GO}$ with $\mathrm{SiO}_{2}$ particles. The obtuse peak which appears between 13 and $23^{\circ}$ can be attributed to the presence of the amorphous silica matrix [69].

XPS was employed as a tool to explore the interactions between GO and $\mathrm{SiO}_{2}$, study the chemical composition composite gels, and confirm the removal of $\mathrm{Na}^{+}$ions. The high resolution XPS spectra of the 0 and 2 wt\% GO integrated gels are presented in Fig. 5. The survey spectra for the $0-2 \mathrm{wt} \% \mathrm{GO}-\mathrm{SiO}_{2}$ gels are also presented, in ESI Fig. 2. XPS analysis indicated the presence of adventitious carbon and an extent of carbon contamination in each of the GO intercalated gels, due to atmospheric carbon or organic deposits residing from the synthetic process [70]. The spectra presented in Fig. 5 indicate little variation within the bonding of the $0,0.5,1$ and $2 \mathrm{wt} \% \mathrm{GO}$ intercalated gels was revealed, as is also evidenced by FTIR. Specifically, the deconvoluted C1s spectra of the pure $\mathrm{SiO}_{2}$ gel and the composite gel with the greatest $\mathrm{GO}$ loading exhibit three functional groups, consistent with both materials (a) $\mathrm{C}=\mathrm{C}$ (b) $\mathrm{C}-\mathrm{OH}$ and (c) $\mathrm{C}-\mathrm{O}$. The data displayed in Table 2, however, reveals that although there are no changeable functional, a variation in chemical composition can be observed. As the GO content increased, from 0 to $2 \mathrm{wt} \%$, the atomic \% of carbon increased from 14.53 to 21.16 at\%.

The examination of the physical and structural properties of the as prepared $\mathrm{GO}-\mathrm{SiO}_{2}$ aerogels indicate that the incorporation of $\mathrm{GO}$ within the silica matrix can reduce pore collapse and thus volume shrinkage without diminishing the intricate properties associated with silica aerogels. The various loadings ( $0.5-2 \mathrm{wt} \%)$ of graphene oxide, deposited amongst the silica particles, did not disrupt the chemical framework of the $\mathrm{SiO}_{2}$ aerogels, excluding slight variation in $\mathrm{Si}-\mathrm{GO}$ bonding as the $\mathrm{GO}$ content of each of the composite gels increases. However, a variation in physical properties of each of the materials was witnessed. As pore volume increased, upon addition of $0.5 \mathrm{wt} \% \mathrm{GO}$, the density and volume shrinkage of the aerogels decreased from 0.19 to $0.14 \mathrm{~g} / \mathrm{cm}^{3}$ and from 80 to $65 \%$, respectively.

The increase in pore volume and decrease in both density and shrinkage suggest that a lower GO content is recommended for the enhancement of $\mathrm{SiO}_{2}$ aerogels. A quantity as small as $0.5 \mathrm{wt} \%$ of $\mathrm{GO}$ is sufficient to support the structure of the gel during ambient drying, preventing pore collapse and network shrinkage, thus also increasing the surface area of the aerogel $\left(390-700 \mathrm{~m}^{2} / \mathrm{g}\right)$.

Previous reports have highlighted the potential of carbon based materials, such as carbon fibres and nanotubes, etc. to, preventing shrinkage and pore collapse of silica aerogels $[63,71,72]$. However, in contrast to GO, quantities $>0.5 \mathrm{wt} \%$ of the silica aerogel mass were often required to reduce shrinkage of the porous silica framework [71, 72]. Additionally, material properties such as, density, pore diameter and surface area were impeded. The intercalation of the silica aerogels with high density carbon fibres increased the density of the composite gels and distorted pore diameter [63]. Alternatively, the addition of more lightweight carbon microfibers preserved the low density of silica aerogels but the surface areas of the materials were diminished [71].

The current study has therefore revealed that GO can be considered as a promising reinforcing agent for silica aerogels (Scheme 2). The carbon based material is capable of acting as a nanofiller, strengthening the

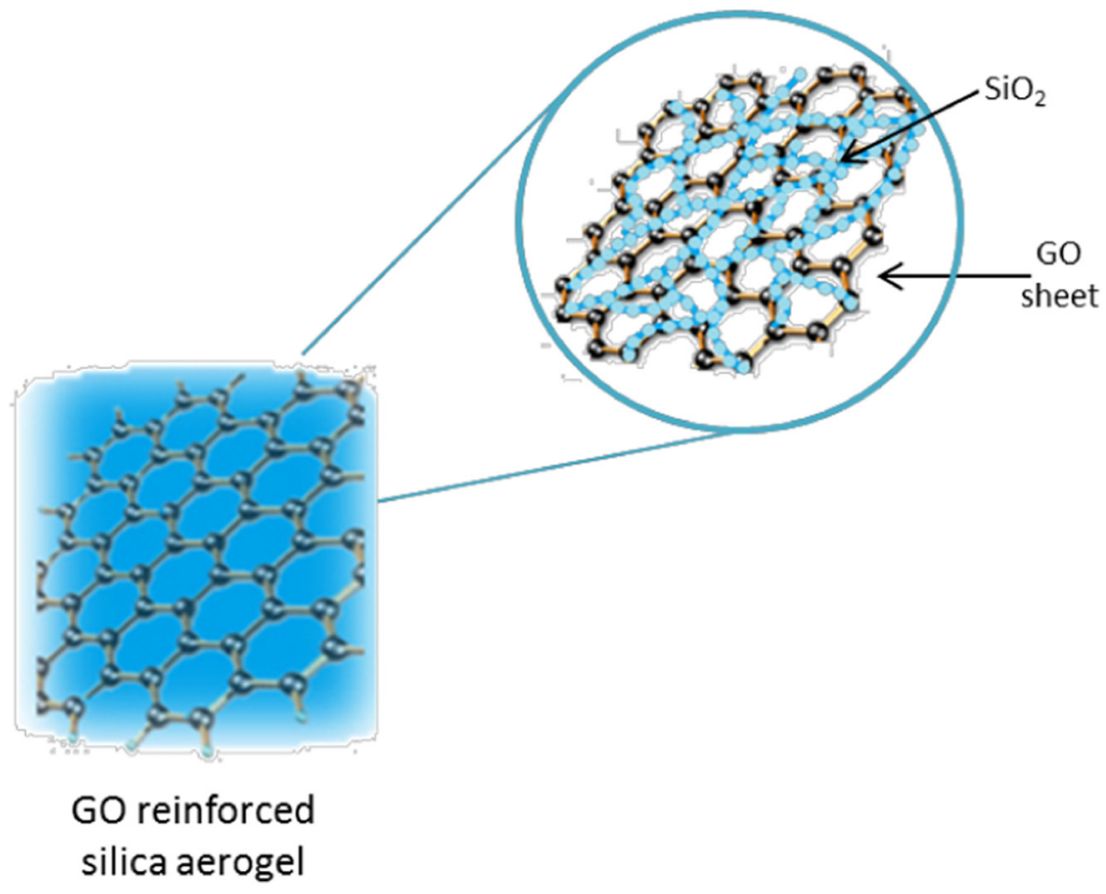

Scheme 2. Graphene oxide reinforced silica aerogel. 
silica backbone while also enhancing the physical properties of the material, such as surface area, pore volume and density.

\section{Conclusions}

Graphene oxide was added to silica aerogels, prepared from sodium silicate, in varying of wt\% contents to investigate the potential of GO to enhance the physical properties of silica aerogels. The study revealed that GO is capable of reducing the volume shrinkage of silica aerogels while simultaneously improving the density, pore volume and surface area of the porous materials.

The incorporation of $\mathrm{GO}$ within the silica matrix enlarged the surface area of each of the aerogels from $390 \mathrm{~m}^{2} / \mathrm{g}$ (pure $\mathrm{SiO}_{2}$ aerogel) to $798 \mathrm{~m}^{2} / \mathrm{g}$ ( $2 \mathrm{wt} \% \mathrm{GO}-\mathrm{SiO}_{2}$ aerogel). Furthermore, the addition of $0.5 \mathrm{wt} \% \mathrm{GO}$ resulted in a 19\% decrease in volume shrinkage, a $26 \%$ decrease in density and a $68 \%$ increase in pore volume. The investigation thus confirmed that the incorporation of small quantities of GO within the silica matrix is not only capable of reinforcing the porous network but also yields aerogels with enhanced properties, such as high surface areas.

\section{Acknowledgements}

The authors would like to acknowledge Dr. Niall McGuinness for useful discussions.

This work was partly supported by the Ministry of Education and Science of the Russian Federation (Grant no. 14.B25.31.0002).

\section{References}

[1] C.A. Pierre, M.G. Pajonk, Chemistry of aerogels and their applications, Chem. Rev. 102 (2002) 4243-4265.

[2] R. Ciriminna, et al., The sol-gel route to advanced silica-based materials and recent applications, Chem. Rev. 113 (8) (2013) 6592-6620.

[3] H. Maleki, Recent advances in aerogels for environmental remediation applications: a review, Chem. Eng. J. 300 (2016) 98-118.

[4] S.S. Kistler, Coherent expanded aerogels and jellies, Nature 127 (1931) 741.

[5] S. Yun, H. Luo, Y. Gao, Superhydrophobic silica aerogel microspheres from methyltrimethoxysilane: rapid synthesis via ambient pressure drying and excellent absorption properties, RSC Adv. 4 (9) (2014) 4535-4542.

[6] H. Schäfer, B. Milow, L. Ratke, Synthesis of inorganic aerogels via rapid gelation using chloride precursors, RSC Adv. 3 (35) (2013) 15263-15272.

[7] S. Kistler, Coherent expanded-aerogels, J. Phys. Chem. 36 (1) (1932) 52-64

[8] S.S. Kistler, Method of producing aerogels, US Patent 2,093,454, 1937.

[9] Graham, T., XXXV.-On the properties of silicic acid and other analogous colloidal substances. J. Chem. Soc., 1864. 17(0): p. 318-327.

[10] G. Nicolaon, S. Teichner, On a new process of preparation of silica xerogels and aerogels and their textural properties, Bull. Soc. Chim. Fr. 5 (1968) 1900.

[11] R. Russo, A. Hunt, Comparison of ethyl versus methyl sol-gels for silica aerogels using polar nephelometry, J. Non-Cryst. Solids 86 (1) (1986) 219-230.

[12] P.H. Tewari, A.J. Hunt, K.D. Lofftus, Ambient-temperature supercritical drying of transparent silica aerogels, Mater. Lett. 3 (9) (1985) 363-367.

[13] Smith, D.M., R. Deshpande, and C. Jeffrey Brinke. Preparation of low-density aerogels at ambient pressure. In MRS Proceedings. 1992. Cambridge Univ Press.

[14] Smith, D.M., et al., Preparation of low-density xerogels at ambient pressure. J. NonCryst. Solids, 1995. 186(0): p. 104-112.

[15] A.E. Gash, et al., New sol-gel synthetic route to transition and main-group meta oxide aerogels using inorganic salt precursors, J. Non-Cryst. Solids 285 (1-3) (2001) 22-28.

[16] A.E. Gash, et al., Use of epoxides in the sol-gel synthesis of porous iron (III) oxide monoliths from Fe (III) salts, Chem. Mater. 13 (3) (2001) 999-1007.

[17] J.L. Mohanan, S.L. Brock, A new addition to the aerogel community: unsupported CdS aerogels with tunable optical properties, J. Non-Cryst. Solids 350 (2004) 1-8.

[18] A.E. Aliev, et al., Giant-stroke, superelastic carbon nanotube aerogel muscles, Science 323 (5921) (2009) 1575-1578.

[19] J. Zou, et al., Ultralight multiwalled carbon nanotube aerogel, ACS Nano 4 (12) (2010) 7293-7302.

[20] X. Zhang, et al., Mechanically strong and highly conductive graphene aerogel and its use as electrodes for electrochemical power sources, J. Mater. Chem. 21 (18) (2011) 6494-6497.

[21] H. Hu, et al., Ultralight and highly compressible graphene aerogels, Adv. Mater. 25 (15) (2013) 2219-2223.

[22] J. Wang, M. Ellsworth, Graphene aerogels, ECS Trans. 19 (5) (2009) 241-247.

[23] N. Leventis, et al., Click synthesis of monolithic silicon carbide aerogels from polyac rylonitrile-coated 3D silica networks, Chem. Mater. 22 (9) (2010) 2790-2803.
[24] P. Aravind, et al., Ambient pressure drying: a successful approach for the preparation of silica and silica based mixed oxide aerogels, J. Sol-Gel Sci. Technol. 54 (1) (2010) 105-117.

[25] S.-W. Hwang, et al., Effective preparation of crack-free silica aerogels via ambient drying, J. Sol-Gel Sci. Technol. 41 (2) (2007) 139-146.

[26] Y. Attia, Sol-Gel Processing and Applications, Springer Science \& Business Media, 2012.

[27] S.D. Bhagat, et al., Textural properties of ambient pressure dried water-glass based silica aerogel beads: one day synthesis, Microporous Mesoporous Mater. 96 (1) (2006) 237-244

[28] S.-W. Hwang, T.-Y. Kim, S.-H. Hyun, Effect of surface modification conditions on the synthesis of mesoporous crack-free silica aerogel monoliths from waterglass via ambient-drying, Microporous Mesoporous Mater. 130 (1-3) (2010) 295-302.

[29] R. Deshpande, et al., Pore structure evolution in silica gel during aging/drying. III. Effects of surface tension, J. Non-Cryst. Solids 144 (1992) 32-44.

[30] J.P. Randall, M.A.B. Meador, S.C. Jana, Tailoring mechanical properties of aerogels for aerospace applications, ACS Appl. Mater. Interfaces 3 (3) (2011) 613-626.

[31] D.Y. Nadargi, A.V. Rao, Methyltriethoxysilane: new precursor for synthesizing silica aerogels, J. Alloys Compd. 467 (1-2) (2009) 397-404

[32] N.D. Hegde, A. Venkateswara Rao, Organic modification of TEOS based silica aerogels using hexadecyltrimethoxysilane as a hydrophobic reagent, Appl. Surf. Sci. 253 (3) (2006) 1566-1572.

[33] S. Cui, et al., Temperature dependent microstructure of MTES modified hydrophobic silica aerogels, Mater. Lett. 65 (4) (2011) 606-609.

[34] D.Y. Nadargi, et al., Studies on rheological properties of methyltriethoxysilane (MTES) based flexible superhydrophobic silica aerogels, Microporous Mesoporous Mater. 117 (3) (2009) 617-626.

[35] Y. Duan, et al., Surface modification and reinforcement of silica aerogels using polyhedral oligomeric silsesquioxanes, Langmuir 28 (43) (2012) 15362-15371.

[36] Maleki, H., L. Durães, and A. Portugal, An overview on silica aerogels synthesis and different mechanical reinforcing strategies. J. Non-Cryst. Solids, 2014. 385(0): p. 55-74.

[37] S. Yun, H. Luo, Y. Gao, Ambient-pressure drying synthesis of large resorcinol-formaldehyde-reinforced silica aerogels with enhanced mechanical strength and superhydrophobicity, J. Mater. Chem. A 2 (35) (2014) 14542-14549.

[38] H. Maleki, L. Duraes, A. Portugal, Development of mechanically strong ambient pressure dried silica aerogels with optimized properties, J. Phys. Chem. C 119 (14) (2015) 7689-7703.

[39] Y. Duan, et al., Self-crosslinkable poly (urethane urea)-reinforced silica aerogels, RSC Adv. 5 (88) (2015) 71551-71558.

[40] H. Maleki, L. Durães, A. Portugal, Synthesis of lightweight polymer-reinforced silica aerogels with improved mechanical and thermal insulation properties for space applications, Microporous Mesoporous Mater. 197 (2014) 116-129.

[41] J.C. Wong, et al., Mechanical and thermal properties of nanofibrillated cellulose reinforced silica aerogel composites, Microporous Mesoporous Mater. 217 (2015) 150-158.

[42] A. Saboktakin, M.R. Saboktakin, Improvements of reinforced silica aerogel nanocomposites thermal properties for architecture applications, Int. J. Biol. Macromol. 72 (2015) 230-234.

[43] J. Fu, et al., Facilitated fabrication of high strength silica aerogels using cellulose nanofibrils as scaffold, Carbohydr. Polym. 147 (2016) 89-96.

[44] A. Sedova, et al., Reinforcing silica aerogels with tungsten disulfide nanotubes, J. Supercrit. Fluids 106 (2015) 9-15.

[45] Z. Li, et al., Aramid fibers reinforced silica aerogel composites with low thermal conductivity and improved mechanical performance, Compos. A: Appl. Sci. Manuf. 84 (2016) 316-325.

[46] Z. Shao, et al., Ambient pressure dried shape-controllable sodium silicate based composite silica aerogel monoliths, Mater. Chem. Phys. 162 (2015) 346-353.

[47] N. Leventis, et al., Nanoengineering strong silica aerogels, Nano Lett. 2 (9) (2002) 957-960.

[48] P. Podsiadlo, et al., Ultrastrong and stiff layered polymer nanocomposites, Science 318 (5847) (2007) 80-83.

[49] O.C. Compton, S.T. Nguyen, Graphene oxide, highly reduced graphene oxide, and graphene: versatile building blocks for carbon-based materials, Small 6 (6) (2010) 711-723.

[50] D. Loche, et al., Incorporation of graphene into silica-based aerogels and application for water remediation, RSC Adv. 6 (71) (2016) 66516-66523.

[51] D.C. Marcano, et al., Improved synthesis of graphene oxide, ACS Nano 4 (8) (2010) 4806-4814.

[52] A.V. Rao, M.M. Kulkarni, Effect of glycerol additive on physical properties of hydrophobic silica aerogels, Mater. Chem. Phys. 77 (3) (2003) 819-825.

[53] A.M. Siouffi, Silica gel-based monoliths prepared by the sol-gel method: facts and figures, J. Chromatogr. A 1000 (1-2) (2003) 801-818.

[54] P. Mezza, J. Phalippou, R. Sempere, Sol-gel derived porous silica films, J. Non-Cryst. Solids 243 (1) (1999) 75-79.

[55] H. Gesser, P. Goswami, Aerogels and related porous materials, Chem. Rev. 89 (4) (1989) 765-788.

[56] A.V. Rao, et al., Reduction in the processing time of doped sodium silicate based ambient pressure dried aerogels using shaker, Microporous Mesoporous Mater. 134 (1-3) (2010) 93-99.

[57] M. Thommes, et al., Physisorption of gases, with special reference to the evaluation of surface area and pore size distribution (IUPAC technical report), Pure Appl. Chem. 87 (9-10) (2015) 1051-1069.

[58] J. Estella, et al., Effects of aging and drying conditions on the structural and textural properties of silica gels, Microporous Mesoporous Mater. 102 (1-3) (2007) 274-282. 
[59] C. Wan, J. Li, Incorporation of graphene nanosheets into cellulose aerogels: enhanced mechanical, thermal, and oil adsorption properties, Appl. Phys. A 122 (2) (2016) 1-7.

[60] S. Bag, et al., Porous semiconducting gels and aerogels from chalcogenide clusters, Science 317 (5837) (2007) 490-493.

[61] Y. Yang, et al., A facile method to fabricate silica-coated carbon nanotubes and silica nanotubes from carbon nanotubes templates, J. Mater. Sci. 44 (17) (2009) 4539-4545.

[62] M. Prassas, et al., Preparation of $\mathrm{xNa}_{2} \mathrm{O}-(1-\mathrm{x}) \mathrm{SiO}_{2}$ gels for the gel-glass process: I. Atmospheric effect on the structural evolution of the gels, J. Non-Cryst. Solids 48 (1) (1982) 79-95.

[63] A. Ślosarczyk, et al., Synthesis and characterization of carbon fiber/silica aerogel nanocomposites, J. Non-Cryst. Solids 416 (2015) 1-3.

[64] S. Dervin, D.D. Dionysiou, S.C. Pillai, 2D nanostructures for water purification: graphene and beyond, Nanoscale (2016).

[65] A.C. Ferrari, J. Robertson, Interpretation of Raman spectra of disordered and amorphous carbon, Phys. Rev. B 61 (20) (2000) 14095.
[66] A. Jorio, et al., Quantum description of Raman scattering, Raman Spectroscopy in Graphene Related Systems 2011, pp. 103-119.

[67] C. Nethravathi, M. Rajamathi, Chemically modified graphene sheets produced by the solvothermal reduction of colloidal dispersions of graphite oxide, Carbon 46 (14) (2008) 1994-1998.

[68] C. Xu, et al., Synthesis of amphiphilic graphite oxide, Carbon 46 (2) (2008) 386-389.

[69] L. Wang, et al., Synthesis of single silica nanotubes in thepresence of citric acid, J. Mater. Chem. 11 (5) (2001) 1465-1468.

[70] M. Connor, C. Colmenares, X-ray photoelectron characterization of $\mathrm{SiO}_{2}$ aerogel, J. Non-Cryst. Solids 201 (1) (1996) 76-80.

[71] A. Ślosarczyk, et al., Synthesis and characterisation of silica aerogel/carbon microfibers nanocomposites dried in supercritical and ambient pressure conditions, J. Sol-Gel Sci. Technol. 76 (1) (2015) 227-232.

[72] T. Sun, et al., Hydrophobic silica aerogel reinforced with carbon nanotube for oils removal, J. Porous. Mater. 21 (6) (2014) 967-973. 